\title{
Adult Rhabdomyosarcoma
}

National Cancer Institute

\section{Source}

National Cancer Institute. Adult Rhabdomyosarcoma. NCI Thesaurus. Code C9130.

An aggressive malignant mesenchymal neoplasm arising from skeletal muscle in adults. 